CAHIERS DE

NARRATOLOGIE

\section{Cahiers de Narratologie}

Analyse et théorie narratives

$14 \mid 2008$

Prose d'idées : formes et savoirs

\title{
Antonio LóPEZ DE VEGA, Paradojas Racionales. En forma de diálogo entre un filósofo y un cortesano, édition, introduction et notes par Máximo Higuera, Madrid, Trifaldi, 2005, $205 \mathrm{p}$.
}

Marie-Laure Acquier

\section{CpenEdition}

Journals

Édition électronique

URL : http://journals.openedition.org/narratologie/617

DOI : 10.4000/narratologie.617

ISSN : 1765-307X

Éditeur

LIRCES

Référence électronique

Marie-Laure Acquier, « Antonio López de VEGA, Paradojas Racionales. En forma de diálogo entre un filósofo y un cortesano, édition, introduction et notes par Máximo Hiquera, Madrid, Trifaldi, 2005, 205 p. », Cahiers de Narratologie [En ligne], 14 | 2008, mis en ligne le 15 juillet 2010, consulté le 22 septembre 2020.

URL : http://journals.openedition.org/narratologie/617; DOI : https://doi.org/10.4000/narratologie.617

Ce document a été généré automatiquement le 22 septembre 2020.

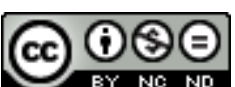

Cahiers de Narratologie - Analyse et théorie narratives est mis à disposition selon les termes de la licence Creative Commons Attribution - Pas d'Utilisation Commerciale - Pas de Modification 4.0 International. 


\title{
Antonio LóPEZ DE VEGA, Paradojas Racionales. En forma de diálogo entre un filósofo y un cortesano, édition, introduction et notes par Máximo Higuera, Madrid, Trifaldi, 2005, 205 p.
}

\author{
Marie-Laure Acquier
}

1 Pour le premier titre de leur nouvelle collection sur l'histoire des idées, les éditions madrilènes Trifaldi nous donnent à lire l'édition établie par Máximo Higuera des Paradoxes Rationnels (Paradojas Racionales), dernier traité en prose écrit par Antonio López de Vega (1586?-1656?). D'origine portugaise, l'auteur passa la majeure partie de sa vie dans le Madrid de la première moitié du XVIIe siècle, sous la protection de hauts personnages de la noblesse. Il a bénéficié comme nombre de poètes et prosateurs de son époque de l'embellie du mécénat sous les règnes successifs de Philippe III et de Philippe IV d'Espagne. Sa dernière œuvre ainsi éditée offre un cas des plus représentatifs de la prose d'idées de la période baroque en Espagne.

2 L'appareil liminaire de l'œuvre retranscrit dans l'édition nous informe que le manuscrit $\mathrm{n}^{\circ}$ 7903, daté de 1654 et conservé à la Bibliothèque Nationale de Madrid, disposait de toutes les pièces nécessaires à sa publication. Il n'avait pourtant jamais été publié du vivant de l'auteur. Le texte avait déjà fait l'objet d'une édition érudite en 1935 de la main d'un passionné d'histoire portugaise, Erasmo Buceta, dans une revue désormais peu accessible (Anejos de la Revista de Filología Española, Anejo XXI, 1935).

Bien que cela ne soit pas lisible dans l'édition qu'il donne -ce que l'on peut déplorer du point de vue de la génétique textuelle et de l'histoire de la censure- Máximo Higuera intègre au texte les rajouts de l'auteur repérables dans le manuscrit ainsi que les phrases probablement barrées par les correcteurs ou censeurs. La graphie et la 
ponctuation sont modernisées pour faciliter la lecture. L'appareil de notes est surtout de nature sémantique. L'élucidation de certaines citations dans le corps du texte manifeste la prédominance des références aux œuvres de Sénèque ; ce qui confirme le rôle de de López de Vega comme vecteur important de la pensée néo-stoicienne en Espagne ${ }^{1}$. Grâce à ce travail, nous disposons donc désormais de l'intégralité d'un texte auparavant réservé aux seuls lecteurs de la Bibliothèque Nationale de Madrid ; un texte au titre singulier qui, comme le rappelle Higuera dans son introduction, a suscité l'intérêt de bien des critiques, historiens de la littérature, historiens des idées ou de la noblesse (José Antonio Maravall, José Luis Abellán, Monroe Hafter par exemple), et plus généralement de tous ceux qui s'intéressent à la prose d'idées dans l'Espagne de Philippe IV.

Outre le résumé de l'œuvre, on trouvera dans l'introduction de Máximo Higuera, une biographie succincte de l'auteur établie à partir des éléments peu fournis -et parfois peu étayés- offerts par la bibliographie classique (ex: l'amitié de l'auteur avec Francisco de Quevedo prouvée par un sonnet), une brève synthèse sur l'état de la question concernant l'œuvre de López de Vega qui mentionnent les critiques espagnols les plus connus, quelques éléments rapides sur la réception de celle-ci au XVIII siècle², qui esquissent le lien entre le baroque et l'époque des Lumières. L'introduction insiste sur le côté peu original des thèmes abordés, très discutés à l'époque il est vrai (opposition entre noblesse héréditaire et noblesse naturelle, signes extérieurs de l'honneur, repoussoir des carrières publiques, violence des armes, cercles de conversation et Académies littéraires où règnent la fatuité et l'ignorance), sur l'originalité de la forme mais aussi sur la démarche qui privilégie une approche rationnelle d'idées communément admises.

5 Si l'on y regarde de plus près, cette œuvre est en effet intéressante à plus d'un titre. Grâce à l'approfondissement de notre connaissance sur les débats contemporains et sur les acteurs politiques au sein de la monarchie espagnole de l'époque moderne, ce qui avait pu être interprété par certains exégètes comme des critiques acerbes de la société du temps nous apparaît plutôt désormais comme un mécanisme de régulation et de maintien de l'équilibre social où chacun participe à un échange d'idées sans remettre en cause le système dont il fait partie ${ }^{3}$. Cette édition des Paradojas Racionales d'Antonio López de Vega permet à ceux qui s'y intéressent d'alimenter leur réflexion sur les notions de consensus social dans la société de l'Espagne moderne.

D'un point de vue formel, l'œuvre revendique dès son titre son appartenance au genre des paradoxes, remis au goût du jour dans la péninsule par la littérature scientifique et morale au XVIe siècle mais elle échappe aussitôt à cette catégorisation par plusieurs aspects. Les six paradoxes de l'œuvre font en effet dialoguer deux personnages type de la cour madrilène, non identifiés, désignés par leur seul statut: un philosophe et un courtisan. Le premier impose une vision raisonnée des hommes, de leurs attitudes et de la société dans laquelle ils évoluent. Le second exprime ses convictions présentées comme issues du sens commun mais qui sont aussitôt défaites par l'argumentaire de son interlocuteur. Les entretiens du philosophe et du courtisan occupent les cinq derniers paradoxes qui reposent sur la confrontation d'idées à partir d'une proposition initiale énoncée en tête de chapitre. Nous sommes dès lors aux confins du traité et du dialogue humaniste ${ }^{4}$. Le premier des six dialogues échappe en outre à cette unité. Qualifié de « narratif » par l'auteur lui-même, il scénarise l'échange de vues par la place qu'il accorde au récit en lui donnant tous les aspects de la fiction. Il installe les 
personnages dans leur décor, les fait se rencontrer puis rendre visite à un troisième qui seul a le privilège d'être nommé : Gerardo. Celui-ci, qualifié de "solitaire à la cour » et qui donne son titre au premier paradoxe, explique les raisons de son éloignement des intrigues courtisanes et de l'agitation de la capitale. Il incarne un idéal de vie retirée aux marges de la cour qui ne manque pas d'originalité. Ce dialogue mis en récit et fictionnalisé confirme le polymorphisme du vaste champ de la prose d'idées dans lequel l'œuvre s'inscrit. Il prouve aussi que la fiction, vecteur de vérité à l'époque concernée au même titre que le traité ou le sermon, ne sert pas de critère discriminant pour établir une séparation factice entre les œuvres appartenant à la prose d'idées et celles qui n'en relèveraient pas.

\section{NOTES}

1. Karl. A. Blüher : Séneca en España. Investigaciones sobre la recepción de Séneca en España desde el siglo XIII hasta el siglo XVII, Madrid, Gredos, 1983 ; Jeremy Robbins, "Scepticism and stoïcism in Spain : Antonio López de Vega's Heraclito y democrito de nuestro siglo", Culture and society in Habsburg Spain : studies presented to R. W. Truman by his pupils and colleagues on the occasion of his retirement, coord. Eric Southworth, Nigel Griffin, Colin P. Thompson, Clive Griffin, Londres, Tamesis, 2001, p. 137-152.

2. Sur cet aspect de la question intéressant à plus d'un titre, on peut consulter Marie-Laure Acquier, "Réflexions sur le lien entre les penseurs baroques et les Lumières. Le cas d'Antonio López de Vega (1586?-1656?)" in Christophe Couderc et Benoît Pellistrandi, "Por discreto y por amigo". Mélanges offerts à Jean Canavaggio, Madrid, Casa de Velásquez, 2005, p. 473-485.

3. Ceci est notamment sensible pour les idées défendues par les arbitristas, ces donneurs d'avis, acteurs économiques ou politiques au sein de la monarchie. Voir Alfredo Alvar Ezquerra, "Dar, ideas, informar y conocer para el rey: el arbitrismo en tiempos de Felipe II", Torre de los Lujanes, 1998, pp. 87-106; Elena García Guerra, Moneda y arbitrios: consideraciones del siglo XVII, Madrid, CSIC, 2003.

4. Jacqueline Ferreras, Les dialogues espagnols du XVIème siècle ou l'expression littéraire d'une nouvelle conscience, Paris, Didier Erudition, 1985.

\section{AUTEUR}

\section{MARIE-LAURE ACQUIER}

Agrégée d'Espagnol, ancien membre de la Casa de Velázquez de Madrid, Marie-Laure Acquier est maître de conférences à l'Université de Nice-Sophia antipolis. 